\title{
Adipokine Mediators of Inflammation and Cardiometabolic Comorbidity in Rheumatoid Arthritis: Is There a Master Adipokine?
}

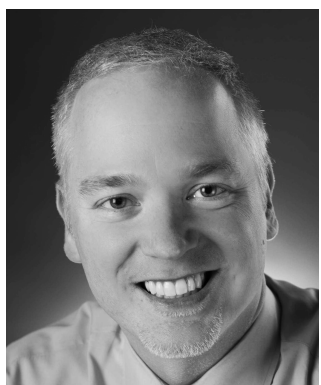

Understanding how immune cells interact with adipocytes to induce the downstream consequences of obesity (i.e., insulin resistance, hepatic steatosis, atherosclerosis, just to name a few) is among the most active areas of current interest among obesity researchers. In fact, that white adipose tissue has secretory capacity is a relatively recent concept. In the early 1990s it was recognized that tumor necrosis factor- $\alpha$ (TNF- $\alpha$ ) and leptin are expressed by adipocytes in proportion to adipose tissue mass and that they possess diverse autocrine and paracrine functions relating to energy metabolism and the neurohormonal regulation of feeding impulses ${ }^{1,2}$. Since then, dozens of secreted adipocyte products have been identified ${ }^{3}$.

That many proteins termed "adipokines" overlap those considered central to the inflammatory pathobiology of rheumatoid arthritis (RA) is of particular interest to RA researchers. Adipocytes secrete inflammatory cytokines [TNF- $\alpha$, interleukin $1 \beta$ (IL-1 $\beta$ ), IL-6], chemokines (monocyte chemoattractant protein-1), acute-phase reactants (serum amyloid A), and others that parallel the expression profiles of inflamed synovium ${ }^{4}$. Adipokine expression is potentiated through activated macrophages and $\mathrm{T}$ lymphocytes within adipose tissue, and represents an additional parallel to the processes occurring in RA synovitis.

Interestingly, many obesity-related complications are also present in patients with RA, even among those who are not obese. Cardiovascular disease (CVD) event rates and atherosclerosis are increased compared with otherwise similar non-RA controls ${ }^{5,6,7}$, and insulin resistance measures are higher in RA, even after controlling for adipose tissue amount, location, and glucocorticoid use. Across many studies, circulating and synovial fluid levels of several adipokines (e.g., leptin ${ }^{8}$, resistin ${ }^{9}$, visfatin ${ }^{10}$, and adiponectin ${ }^{11}$ ) are also higher among RA patients compared with controls. While some of this increase could be related to higher fat mass and/or a tendency to accumulate fat in the visceral compartment in some patients with $\mathrm{RA}^{12}$, it likely does not explain the entire increase in levels. However, the implied pathogenic effects of adipose-derived "hyperadipokinemia" in RA have been difficult to support, particularly as there are seemingly paradoxical associations of adiposity with several key adverse outcomes in RA. For example, several high-quality studies have demonstrated that RA patients with a higher body mass index (BMI, a surrogate for adiposity), have lower all-cause and CVD mortality ${ }^{13}$, and tend to be protected from erosive damage $^{14}$. This protection is found to exist despite the observation that RA patients with higher BMI tend to have higher measures of disease activity than those with lower $\mathrm{BMI}$, and a diminished treatment response to some biologic disease-modifying antirheumatic drugs ${ }^{15}$. Clearly, the relationships that link the inflammatory products of adipose tissue with RA-associated outcomes are complex, dynamic, possibly conditioned on other patient-specific features, and not easily explained using simple correlations and bivariate modeling techniques. The search for an elusive adipokine "master-switch" in RA continues.

Enter chemerin. Chemerin was first described in 2003 as an adipokine that signals through several chemokine receptors ${ }^{16}$. Chemerin levels have been shown to be higher in several chronic inflammatory diseases, such as inflammatory bowel disease, psoriasis, systemic lupus erythematosus, and multiple sclerosis ${ }^{17}$. TNF- $\alpha$ induces adipocytes to secrete chemerin, which in turn has been shown to upregulate the production of TNF- $\alpha$, IL-6, IL-1 $\beta$, and chemokine ligand 2 in both human chondrocytes and synoviocytes $^{18}$. Relevant to this, synovial fluid concentrations of chemerin are elevated in osteoarthritis, psoriatic arthritis, and RA relative to circulating levels, suggesting that the joint may be the site of production ${ }^{17}$. Downstream, chemerin may affect insulin sensitivity and promote atherogenesis. Insulin-stimulated glucose uptake into skeletal muscle was decreased upon treatment with chemerin ${ }^{19}$;

See Chemerin and CVD risk in RA, page 1746

Personal non-commercial use only. The Journal of Rheumatology Copyright (C) 2014. All rights reserved 
however, this effect has not been shown in all studies. In addition, chemerin is expressed in macrophage foam cells of atherosclerosis in proportion to lesion $\operatorname{size}^{20}$. Taken together, it is compelling to implicate chemerin as a potential link between articular inflammation and cardiometabolic comorbidities.

In this issue of The Journal, Dessein and colleagues present a cross-sectional analysis of the association of chemerin with cardiometabolic intermediates, RA characteristics, and ultrasonographic measures of atherosclerosis in the common carotid artery ${ }^{21}$. They report that higher circulating chemerin levels were associated with several RA characteristics, including measures of disease activity. Higher chemerin levels were associated with CVD measures only among certain subgroups. Chemerin was associated with circulating markers of endothelial activation only among patients with traditional CVD risk factors, with common carotid intima-medial thickness only among those who were obese or met criteria for the metabolic syndrome, and seemingly counter-intuitively with carotid plaque only among those who were not obese. While these findings fall short of establishing causality, they are hypothesis-generating and provide support for subsequent longitudinal and mechanistic studies.

In designing those followup studies, several important issues are of particular interest. The first is specificity: Does circulating chemerin induce the same effect in RA as it does in healthy controls or even in other non-RA chronic inflammatory conditions? This is particularly relevant for the design and implementation of RA-specific intervention strategies should chemerin prove to be a therapeutic target.

The second is the source of chemerin in RA. Does the excess chemerin in RA driving pathogenic downstream effects originate in inflamed synovium, or is it being produced in greater than expected excess by white adipose tissue, possibly itself stimulated by the systemic inflammation of RA? This has relevance to potential therapeutic targets and is hinted at in the present report, because chemerin was associated with several of the studied CVD outcomes only among those who were obese.

A third issue relates to possible confounders. Adipocytes produce many adipokines in response to inflammatory stimuli. These tend to be highly correlated, and exploring the independent effects of a single adipokine requires eliminating the confounding effects of the many that are upregulated in parallel and that may be carrying the "true" effect. This is quite challenging in the setting of an observational study. While Dessein and colleagues considered several of the most prominent adipokines and adjusted for the most relevant to the analysis (e.g., leptin), there remains a possibility that the effects they observed are due not to chemerin but to a closely associated factor or factors.

Despite these concerns, the work of Dessein and colleagues provides a compelling new springboard for investigation, one in which the elusive adipokine "master-switch" may be discovered.

JON T. GILES, MD, MPH,

Division of Rheumatology,

Columbia University,

College of Physicians and Surgeons,

Physicians \& Surgeons Building, Suite 10-445, 630 W 168th St.,

New York, New York 10032, USA.

Address correspondence to Dr. Giles; E-mail: jtg2122@columbia.edu

\section{REFERENCES}

1. Hotamisligil GS, Shargill NS, Spiegelman BM. Adipose expression of tumor necrosis factor-alpha: direct role in obesity-linked insulin resistance. Science 1993;259:87-91.

2. Chua SC Jr, Chung WK, Wu-Peng XS, Zhang Y, Liu SM, Tartaglia $\mathrm{L}$, et al. Phenotypes of mouse diabetes and rat fatty due to mutations in the OB (leptin) receptor. Science 1996;271:994-6.

3. Poulos SP, Hausman DB, Hausman GJ. The development and endocrine functions of adipose tissue. Mol Cell Endocrinol 2010;323:20-34

4. Ouchi N, Parker JL, Lugus JJ, Walsh K. Adipokines in inflammation and metabolic disease. Nat Rev Immunol 2011; 11:85-97.

5. Avina-Zubieta JA, Choi HK, Sadatsafavi M, Etminan M, Esdaile JM, Lacaille D. Risk of cardiovascular mortality in patients with rheumatoid arthritis: a meta-analysis of observational studies. Arthritis Rheum 2008;59:1690-7.

6. Giles JT, Szklo M, Post W, Petri M, Blumenthal RS, Lam G, et al. Coronary arterial calcification in rheumatoid arthritis: comparison to the multi-ethnic study of atherosclerosis. Arthritis Res Ther 2009; 11:R36.

7. Kobayashi H, Giles JT, Polak JF, Blumenthal RS, Leffell MS, Szklo $\mathrm{M}$, et al. Increased prevalence of carotid artery atherosclerosis in rheumatoid arthritis is artery-specific. J Rheumatol 2010;37:730-9.

8. Tian G, Liang JN, Pan HF, Zhou D. Increased leptin levels in patients with rheumatoid arthritis: a meta-analysis. Ir J Med Sci 2014 Feb 9 (E-pub ahead of print).

9. Senolt L, Housa D, Vernerova Z, Jirasek T, Svobodova R, Veigl D, et al. Resistin in rheumatoid arthritis synovial tissue, synovial fluid and serum. Ann Rheum Dis 2007;66:458-63.

10. Otero M, Lago R, Gomez R, Lago F, Dieguez C, Gomez-Reino JJ, et al. Changes in plasma levels of fat-derived hormones adiponectin, leptin, resistin and visfatin in patients with rheumatoid arthritis. Ann Rheum Dis 2006;65:1198-201.

11. Senolt L, Pavelka K, Housa D, Haluzik M. Increased adiponectin is negatively linked to the local inflammatory process in patients with rheumatoid arthritis. Cytokine 2006;35:247-52.

12. Giles JT, Allison M, Blumenthal RS, Post W, Gelber AC, Petri M, et al. Abdominal adiposity in rheumatoid arthritis: association with cardiometabolic risk factors and disease characteristics. Arthritis Rheum 2010;62:3173-82.

13. Escalante A, Haas RW, del Rincon I. Paradoxical effect of body mass index on survival in rheumatoid arthritis: role of comorbidity and systemic inflammation. Arch Intern Med 2005;165:1624-9.

14. Westhoff G, Rau R, Zink A. Radiographic joint damage in early rheumatoid arthritis is highly dependent on body mass index. Arthritis Rheum 2007;56:3575-82.

15. Heimans L, van den Broek M, le Cessie S, Siegerink B, Riyazi N, $\mathrm{Han} \mathrm{KH}$, et al. Association of high body mass index with decreased treatment response to combination therapy in recent-onset rheumatoid arthritis patients. Arthritis Care Res 2013;65:1235-42. 
16. Wittamer V, Franssen JD, Vulcano M, Mirjolet JF, Le Poul E, Migeotte I, et al. Specific recruitment of antigen-presenting cells by chemerin, a novel processed ligand from human inflammatory fluids. J Exp Med 2003;198:977-85.

17. Gomez R, Conde J, Scotece M, Gomez-Reino JJ, Lago F, Gualillo $\mathrm{O}$. What's new in our understanding of the role of adipokines in rheumatic diseases? Nat Rev Rheumatol 2011;7:528-36.

18. Kaneko K, Miyabe Y, Takayasu A, Fukuda S, Miyabe C, Ebisawa $\mathrm{M}$, et al. Chemerin activates fibroblast-like synoviocytes in patients with rheumatoid arthritis. Arthritis Res Ther 2011;13:R158.

19. Sell H, Laurencikiene J, Taube A, Eckardt K, Cramer A, Horrighs $\mathrm{A}$, et al. Chemerin is a novel adipocyte-derived factor inducing insulin resistance in primary human skeletal muscle cells. Diabetes 2009;58:2731-40.
20. Kostopoulos CG, Spiroglou SG, Varakis JN, Apostolakis E, Papadaki HH. Chemerin and CMKLR1 expression in human arteries and periadventitial fat: a possible role for local chemerin in atherosclerosis? BMC Cardiovasc Disord 2014;14:56.

21. Dessein PH, Tsang L, Woodiwiss AJ, Norton GR, Solomon A. Circulating concentrations of the novel adipokine chemerin are associated with cardiovascular disease risk in rheumatoid arthritis. J Rheumatol 2014;41:1746-54.

J Rheumatol 2014;41:1725-7; doi:10.3899/jrheum.140856 